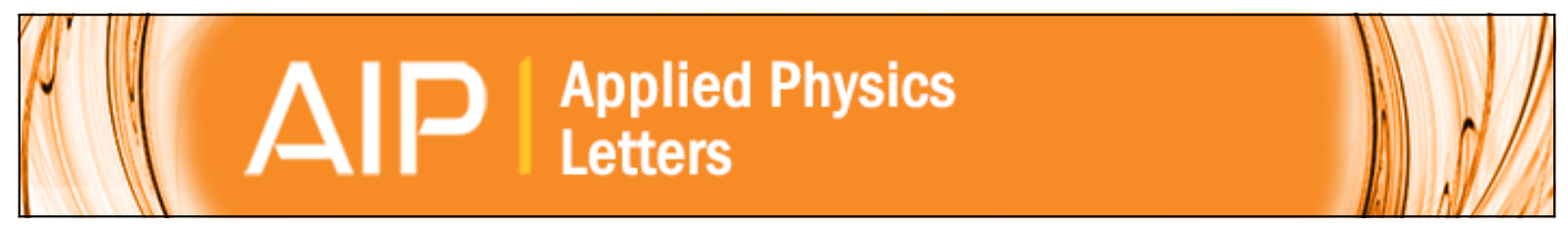

\title{
Second harmonic generation in a photonic crystal
}

Jordi Martorell, R. Vilaseca, and R. Corbalán

Citation: Applied Physics Letters 70, 702 (1997); doi: 10.1063/1.118244

View online: http://dx.doi.org/10.1063/1.118244

View Table of Contents: http://scitation.aip.org/content/aip/journal/apl/70/6?ver=pdfcov

Published by the AIP Publishing

\section{Instruments for advanced science}
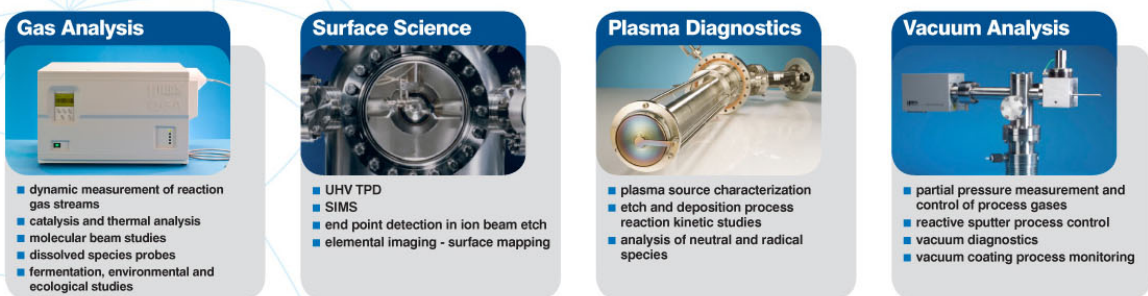

contact Hiden Analytical for further details

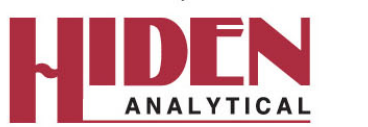

info@hideninc.com www.HidenAnalytical.com CLICK to view our product catalogue 


\title{
Second harmonic generation in a photonic crystal
}

\author{
Jordi Martorella) \\ Departament de Física, Universitat Autònoma de Barcelona, 08193 Bellaterra, Barcelona, Spain \\ R. Vilaseca \\ Departament de Física i Enginyeria Nuclear, Universitat Politècnica de Catalunya, 08222 Terrassa, \\ Barcelona, Spain \\ R. Corbalán \\ Departament de Física, Universitat Autònoma de Barcelona, 08193 Bellaterra, Barcelona, Spain
}

(Received 4 October 1996; accepted for publication 9 December 1996)

\begin{abstract}
Phase matched second harmonic generation is observed experimentally in a centrosymmetric crystalline lattice of dielectric spheres of optical dimensions. The inversion symmetry is broken locally at the surface of each sphere in such a way that the scattered second harmonic light interferes constructively leading to a nonvanishing macroscopic field. Phase matching of the fundamental and second harmonic waves in such periodic lattice is observed to be naturally provided by the bending of the photon dispersion curve at the edge of the Bragg reflection band of a given set of lattice planes. (C) 1997 American Institute of Physics. [S0003-6951(97)03706-6]
\end{abstract}

By the end of the last decade attention was drawn to materials with a 3-dimensional (3d) periodic distribution of the dielectric constant or photonic band gap materials, ${ }^{1}$ where it would be possible to control the photons to be emitted by atoms or molecules embedded in them. ${ }^{1,2}$ Although most of the effort in the field focused on the search of a material that exhibits a full photonic band gap, it has been recognized that nonlinear optical effects may play an important role in the development of more efficient optoelectronic devices $^{3}$ that rely on the special properties of materials that exhibit a photonic band gap only in selected directions, such as colloidal crystals. ${ }^{4}$ In this letter, we report experimental results that show how the scattered light at the double frequency from the surface of small spherical particles that self organize in a crystalline lattice, ${ }^{5}$ are used as a new mechanism for second harmonic generation. The long range periodic order of this structure provides, in a natural manner, the necessary phase matching of the incident laser beam and the generated beam at the double frequency. In such nonlinear colloidal crystals, the nonlinearity of the molecule is not linked to the phase matching mechanism, giving an enormous flexibility for the application of these nonlinear photonic crystals in the implementation of optoelectronic devices, as well as in the study of surface chemical processes in a macroscopic sample.

Efficient second harmonic generation (SHG) has been mostly limited to noncentrosymmetric crystals that must be also birefringent, providing the phase matching mechanism necessary for a continuous growth of the wave at the double frequency. However, SHG in the dipole approximation, a process forbidden in the bulk of centrosymmetric crystals, would be allowed in a photonic crystal composed of polystyrene spherical particles of optical dimensions ordered in an unsheared lattice, because of the local breaking of the inversion symmetry at the surface of each spherical particle. As is well known, second harmonic (SH) light may be reflected

\footnotetext{
${ }^{a}$ Current address: Departament of Física i Enginyeria Nuclear, Universitat Politècnica de Catalunya, 08222 Terrassa, Barcelona, Spain.

Electronic mail: martorell@fen.upc.es
}

from the interface of two centrosymmetric media, due to the lack of inversion symmetry of the surface layers, ${ }^{6}$ or scattered by orientational fluctuations of molecules that locally break the macroscopic centrosymmetry of a solution. ${ }^{7}$ In our experiments, to enhance the second order nonlinear interaction present at this boundary, we adsorbed a layer of strongly nonlinear molecules on the surface of each sphere, of a colloidal crystal ordered in a face centered cubic (fcc) lattice. ${ }^{5}$ Stable aqueous suspensions of negatively charged microspheres were coated by dialysis with a positively charged chromophore of a dye molecule with a high nonlinear coefficient. The negative surface charge of each sphere as well as the hydrophobic character of sulfate latex microspheres help the formation of a surface layer of nonlinear molecules with a preferred orientation of the permanent dipole moment. In our experiments we coated 0.115 - $\mu$ m-diam spheres from Interfacial Dynamics Corporation with the chromophore part of the dye molecule. The polystyrene suspension was dialyzed overnight in a $2 \times 10^{-5} \mathrm{M}$ aqueous solution of Malachite Green. With the colloidal suspension of dye coated spheres a single fcc crystal was formed in a $1 \mathrm{~mm}$ path length cell. The colloidal suspension was added to a cell $(50 \times 10 \times 1 \mathrm{~mm})$ containing a mixed bed ion exchange resin in the bottom. After three or four days a single crystal forms as stray ions in solution diffuse to the resin.

For continuous growth of the $\mathrm{SH}$ field intensity, in addition to a spatially nonuniform response to the nonlinear interaction, it is necessary to phase match the fundamental and SH beams. Pioneering work by Lawandy et al. ${ }^{4}$ proposed the use of the birefringence exhibited by sheared colloidal crystals as the phase matching mechanism for nonlinear photonic crystals. However, in periodic material such as an unsheared crystalline lattice, the mechanism of phase matching can be naturally provided by the bending of the photon dispersion curve at the boundary of the forbidden zone or Bragg reflection band. ${ }^{8}$ Numerical calculations predict a maximum SHG in reflection or transmission for modes outside but near the forbidden zone. ${ }^{9}$ Propagation of light at the smaller angle side of the Bragg reflection band corresponding to the (111) planes of the fcc lattice results in the appropriate change in 


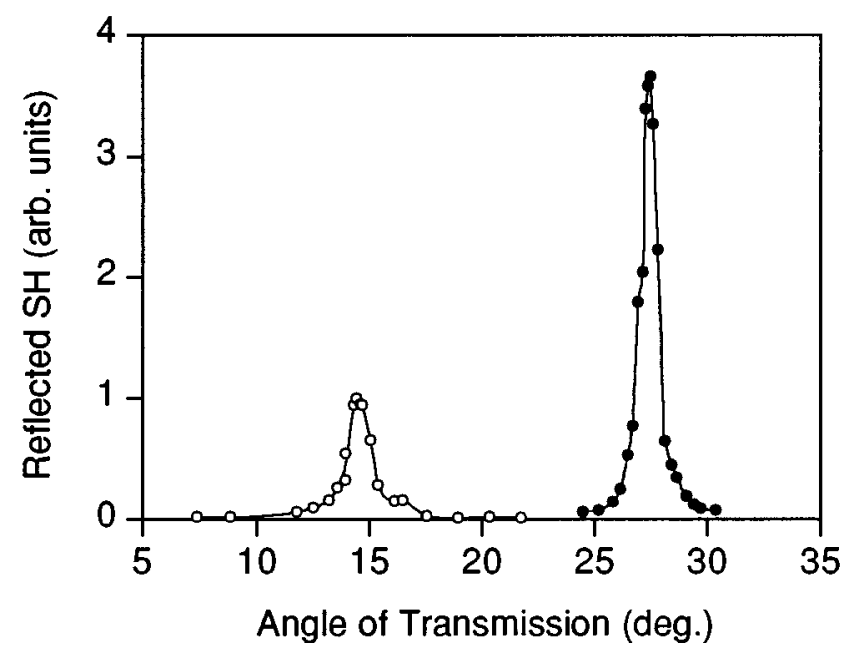

FIG. 1. Intensity of the second harmonic light generated inside a single colloidal crystal, measured in a reflection geometry as a function of the angle of the transmitted fundamental beam relative to the normal of the (111) planes. The open circles correspond to a high sphere concentration crystal, while the solid dots correspond to a $25 \%$ lower sphere concentration crystal. The wavelength of the incident laser pulse was $1064 \mathrm{~nm}$. Single crystals were formed in two $1 \mathrm{~mm}$ path length cuvettes from two different concentrations of $0.115 \mu \mathrm{m}$ in diameter polystyrene spheres coated with Malachite Green in an aqueous suspension. Both lines connecting the experimental data points are a guide for the eye.

effective index of refraction for the wavelength of the SH field. Since the nonlinear material is localized on the surface of every sphere of each (111) plane, and there is no nonlinear material in between planes, phase matching is from plane to plane. This separation between the (111) planes can be tuned to the desired wavelength, previous to the crystal formation, by concentrating the suspension in a dialysis prepared to remove the adequate amount of water from the colloidal suspension.

The prepared sample was excited using an active-passive mode-locked laser emitting 35 ps pulses in the near infrared, at a wavelength of $1064 \mathrm{~nm}$. The laser pulses of an average energy of $<5 \mathrm{~mJ}$ were focused down to a spot size $2 \mathrm{~mm}$ in diameter at the surface of the sample. The polarization of the incident beam was set to be parallel to the plane of incidence, and the polarization of the generated $\mathrm{SH}$ beam was selected using a Glann-Laser polarizer. The SH intensity was detected using a R212 Hamamatsu photomultiplier tube after passage through a heat absorbing filter and an interference filter centered at $532 \mathrm{~nm}$. The wavelength of the generated beam was determined to be $532 \mathrm{~nm}$ using a monochromator with a resolution of $0.1 \mathrm{~nm}$. Measurements of the reflected SH intensity when both the exciting field and the generated field at the double frequency were polarized parallel to the plane of incidence are shown in Fig. 1 as a function of the angle of the transmitted fundamental beam relative to the normal of the (111) planes for two fcc crystals, corresponding to two different sphere concentrations. At a low sphere concentration a peak of maximum SH intensity is observed at $27.4^{\circ}$, while at a higher concentration the peak is shifted down to $14.5^{\circ}$. The $p$-polarized $\mathrm{SH}$ field intensity was measured to be 500 times larger than the intensity for the SH $s$-polarized field. Although similar results were obtained in transmission, maximum SHG in transmission is 0.3

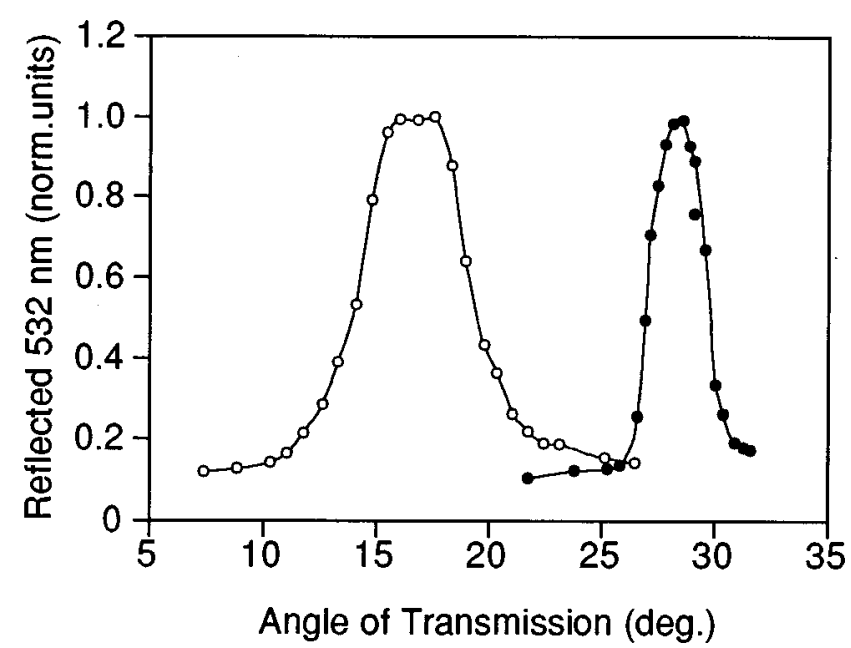

FIG. 2. Reflection of incident light at $532 \mathrm{~nm}$ as a function of the angle of the transmitted beam relative to the normal of the (111) planes for the same crystals considered in Fig. 1. The open circles correspond to the high sphere concentration crystal, while the solid dots correspond to the low sphere concentration crystal of Fig. 1. The reflected intensity is normalized in the figure to the reflected intensity at the angle satisfying the Bragg condition, that was measured to be on average $93 \%$. Both lines connecting the experimental data points are a guide for the eye.

times the maximum peak for SHG in reflection; a reflection geometry is considered to simplify the analysis. In such a geometry interference with the SH light generated in the bulk of the cell walls is strongly reduced. Similar measurements were taken from a crystal of uncoated spheres at the high concentration, and determined that the Malachite Green molecules coating the sphere surface produce an enhancement of the second order nonlinear interaction by approximately one order of magnitude.

Experimental measurements of the passive properties of the colloidal crystal used were performed to determine the origin of the phase matching mechanism involved in the SHG process. Light at $532 \mathrm{~nm}$, obtained by doubling the frequency of the laser pulse in a potassium dihydrogen phosphate (KDP) crystal, was used to measure the reflectivity of the colloidal crystal at the frequency of the SH wave. The reflection from the (111) planes of both fcc colloidal crystals as a function of the angle of the transmitted beam relative to the normal of the (111) planes at a wavelength of $532 \mathrm{~nm}$ is shown in Fig. 2. Maximum reflection occurs when the Bragg condition $2 \mathrm{~d} \cos \theta=\lambda / n$ is satisfied at $28.3^{\circ}$ for the low sphere concentration crystal and at $16.7^{\circ}$ for the crystal with a higher sphere concentration. As the density of spheres increases the angle satisfying the Bragg condition becomes smaller in proportion to the amount of water removed from the suspension. ${ }^{5}$ Notice by comparing Figs. 1 and 2 that, maximum $\mathrm{SH}$ is always generated at the smaller angle side of the corresponding crystal stop band shown in Fig. 2. This is consistent with a phase matching of the fundamental and $\mathrm{SH}$ beams due to a decrease of roughly 0.02 in the effective index of refraction at the left edge of the top band. This change in the effective index of refraction for a wave propagating at the $\mathrm{SH}$ frequency is sufficient to overcome the phase lag between the fundamental and $\mathrm{SH}$ waves introduced by the normal dispersion of water. ${ }^{10} \mathrm{By}$ comparing the maximum power conversion of a crystal $100 \mu \mathrm{m}$ thick and the 
maximum conversion at $27.4^{\circ}$ shown in Fig. 1 for the $1 \mathrm{~mm}$ crystal, we can establish the dependence of the $\mathrm{SH}$ power conversion with the length of the crystal. This dependence should be quadratic in the case of perfect phase matching. However, in a nonperfect crystal actual power conversion is approximately proportional to $L^{2 *} \exp (-\alpha L)$, where $L$ is the length of the crystal and $\alpha$ the extinction coefficient. Scattering losses introduced by defects in the crystalline lattice, in addition to the non-negligible absorption cross section at $532 \mathrm{~nm}$ of Malachite Green, result in an extinction coefficient that was measured at normal incidence to be 28 $\mathrm{cm}^{-1}$ for the low sphere concentration crystal. Using this value for the extinction coefficient in the expression above, a conversion efficiency ratio of 8 is predicted when comparing a $1 \mathrm{~mm}$ and a $100-\mu \mathrm{m}$-thick crystal, in close agreement with the experimentally measured power conversion at the peak of maximum SHG shown in Fig. 1 to be six times larger than in a $100-\mu \mathrm{m}$-thick crystal. The same quadratic behavior is observed in experiments performed using spheres with a smaller diameter and consequently with a lower extinction coefficient of $21 \mathrm{~cm}^{-1}$. In such a case the experimentally observed power conversion ratio when comparing a $1 \mathrm{~mm}$ and a $100-\mu \mathrm{m}$-thick crystal was 9.5 , in close agreement to the predicted value of 14.5 .

This second order nonlinear process at the bulk of a centrosymmetric colloidal crystal is attributed to the nonlinear molecules adsorbed on each sphere. Contribution to the global SH field from each sphere in the crystalline lattice may be accounted for by using a simple theoretical model that considers in a first step the light scattered from the surface of each sphere in the Rayleigh-Gans regime assuming that the permanent dipole moment of the molecule is oriented on average in the radial direction defined from the center of each sphere. Rayleigh-Gans scattering is a valid approximation in the present case, since the ratio of the dielectric constant of the sphere relative to the dielectric constant of the surrounding medium is close to unity. ${ }^{11}$ In consistency with a nonlinearity localized at the surface of each sphere is the decrease in reflected SH light when the angle of reflection approaches zero, ${ }^{12}$ seen in Fig. 1 by comparing the relative height of the two peaks of maximum SHG.

At present, scattering losses introduced by defects in the crystalline lattice limit the conversion efficiency between $10^{2}$ and $10^{3}$ times that of a single layer with the same surface density of Malachite Green adsorbed on a glass plate. However, the particular configuration of the SHG mechanism described sets no limits to the conversion efficiency other than limitations of technical character, such as increasing the density and thickness of the nonlinear molecule coating layer (possibly through a process of chemical adsorption), increasing the nonlinearity of the molecule itself, and the overall quality of the colloidal crystal.

In conclusion, we have observed a mechanism for SHG, attributed to the scattering of SH light from the surface of spherical particles of optical dimensions, when these particles are ordered in a crystalline lattice. We have also demonstrated phase matching due to the long range periodic distribution of dielectric material in a three-dimensional lattice of latex microspheres suspended in water. In this new mechanism of SHG, the nonlinear susceptibility is not dictated by the properties of a birefringent crystal, since the nonlinearity in the molecule is independent of the phase matching mechanism that is provided by the existing periodicity inherent of a photonic crystal. This offers a wide range of possibilities in selecting the most adequate nonlinear molecule. Note also, that charged polystyrene particles self organize in the three-dimensional long range periodic lattice, avoiding the use of expensive and time consuming techniques of multilayer deposition. On the other hand, applications of photonic crystalline lattices are not limited to efficient SHG; the flexibility in selecting the nonlinear molecule makes the nonlinear photonic crystal particularly attractive in the study of surface chemical processes, and in the implementation and improvement of nonlinear optoelectronic devices. Finally, the observation of the scattering of second harmonic light from small spherical particles opens up new possibilities for research in the basic field of propagation of electromagnetic waves in ordered and disordered media.

${ }^{1}$ E. Yablonovitch, Phys. Rev. Lett. 58, 2059 (1987); J. Maddox, Nature (London) 348, 481 (1990); E. Yablonovitch and K. M. Leung, Nature (London) 351, 278 (1991).

${ }^{2}$ J. Martorell and N. M. Lawandy, Phys. Rev. Lett. 65, 1877 (1990).

${ }^{3}$ M. Scalora, J. P. Dowling, C. M. Bowden, and M. J. Bloemer, Phys. Rev. Lett. 73, 1368 (1994).

${ }^{4}$ N. M. Lawandy, S. A. Johnston, and J. Martorell, Opt. Commun. 65, 425 (1988).

${ }^{5}$ P. A. Hiltner and I. M. Krieger, J. Chem. Phys. 73, 2386 (1969); R. Williams and R. S. Crandall, Phys. Rev. A 48, 225 (1974); R. J. Carlson and S. A. Asher, Appl. Spectrosc. 38, 297 (1984).

${ }^{6}$ F. Brown and M. Matsuoka, Phys. Rev. 185, 985 (1969); T. F. Heinz, C. K. Chen, D. Ricard, and Y. R. Shen, Phys. Rev. Lett. 48, 478 (1982).

${ }^{7}$ K. Clays and A. Persoons, Phys. Rev. Lett. 66, 2980 (1991).

${ }^{8}$ N. Bloembergen and A. J. Sievers, Appl. Phys. Lett. 17, 483 (1970); J. P. van der Ziel and M. Ilegems, ibid. 28, 437 (1976); A. Yariv and P. Yeh, J. Opt. Soc. Am. 67, 438 (1977).

${ }^{9}$ J. Martorell and R. Corbalán, Opt. Commun. 108, 319 (1994).

${ }^{10}$ I. Thormhälen, J. Straub, and U. Grigull, J. Phys. Chem. Ref. Data 14, 933 (1985).

${ }^{11}$ See, for example, H. C. Van de Hulst, Light Scattering by Small Particles (Dover, New York,1981).

${ }^{12}$ R. W. J. Hollering and W. J. O. V. Teesselink, Opt. Commun. 79, 224 (1990) 